\title{
CALCULATION OF THE \\ THERMODYNAMICAL POTENTIAL FOR THE ELECTRON-IONIC MODEL OF METAL WITH NONLOCAL INTERACTIONS
}

\author{
P.M.YAKIBCHUK \\ Lviv Ivan Franko State University \\ Department for Theoretical Physics \\ 12 Dragomanov St., UA-290005 Lviv-05, Ukraine
}

Received February 25, 1997

\begin{abstract}
The thermodynamical potential of the electron-ionic model of nontransition metals with a two-particle nonlocal interaction is presented in terms of the peturbation theory with respect to $n$ - particle static correlation functions and $n$-particle polarization operators of the homogeneous electronic liquid.
\end{abstract}

\section{Introduction}

There are two approaches for description of the electron-ionic interactions in the modern microscopic theory of nontransition metals. The first one is based on the theory of pseudopotentials (or model potentials) $[1,2]$ and the second is based one on the reduction of the statistical operator of the precise electron-nuclear model [3,4]. In both approaches there appears the Hamiltonian of the electron-ionic model with effective nonlocal interactions: in the first approach they are pair-wise whereas in the second approach they are $n$-particle $(n \geqslant 2)$. To simplify the problem we can neglect nonimportant many-particle interactions when $n \geqslant 3$. Using the representation of secondary quantization for the electron subsystem as well as the coordinate representation for the ionic one, let us express the Hamiltonian of the electron-ionic model in the standard form:

$$
\hat{H}=\hat{H}_{e}+\hat{H}_{e i}+\hat{H}_{i},
$$

where

$$
\begin{aligned}
& \hat{H}_{e}=\hat{H}_{0}+\hat{V}_{e e}, \quad \hat{H}_{0}=\sum_{\vec{k}, s} \varepsilon_{\vec{k}} C_{\vec{k}, s}^{+} C_{\vec{k}, s}, \\
& \hat{V}_{e e}=(2 V)^{-1} \sum_{\vec{q} \neq 0} V_{q} \sum_{\vec{k}_{1}, \vec{k}_{2}} \sum_{s_{1}, s_{2}} C_{\vec{k}_{1}+\vec{q}, s_{1}}^{+} C_{\vec{k}_{2}-\vec{q}, s_{2}}^{+} C_{\vec{k}_{2}, s_{2}} C_{\vec{k}_{1}, s_{1}}
\end{aligned}
$$

is the Hamiltonian of the electron liquid model,

$$
\hat{H}_{e i}=N_{i} V^{-1} \sum_{\vec{q}} S_{-\vec{q}} \sum_{\vec{k}, s} W_{0}(\vec{k}+\vec{q}, \vec{k}) C_{\vec{k}+\vec{q}, s}^{+} C_{\vec{k}, s}
$$

(C) P.M.Yakibchuk, 1997

ISSN 0452-9910. Condensed Matter Physics 1997 No 10 (179-187) 179 
is the operator of the nonlocal electron-ionic interaction; $\hat{H}_{i}(R)-$ is the Hamiltonian of the ionic subsystem. The formulae (2)-(3) contain the following notations: $\varepsilon_{k}=\hbar^{2} k^{2} / 2 m, V_{q}=4 \pi e^{2} q^{-2}$ is the Fourier transform of the Coulomb potential; $V$ is the volume of the system which contains $N$ electrons and $\left(N_{i}=Z^{-1} N\right)$ ions. $C_{\vec{k}, s}^{+}, C_{\vec{k}, s}$ are the operators of creation and annihilation of the electrons in the plane wave basis; $s= \pm 1 / 2$ are the spin variables,

$$
S_{\vec{q}}=N_{i}^{-1} \sum_{j=1}^{N_{i}} \exp \left(i \vec{q} \vec{R}_{j}\right)
$$

is the stuctural factor of the ionic subsystem. In both approaches mentioned above the formfactor $W$ possesses the similar cover structure [4-6].

$$
W_{0}(\vec{k}+\vec{q}, \vec{k})=\sum_{l=0}^{l_{0}} a_{l}(\vec{k}+\vec{q}, \vec{k})+\sum_{l_{1} \neq l_{2}=0}^{l_{0}} b_{l_{1} l_{2}}(\vec{k}+\vec{q}, \vec{k})+\ldots
$$

where the summarizing is perfomed over to the orbital quantum numbers of the occupied electronic states in the internal covers of the ions.

It is convenient to separate the nonlocal part in the $W_{0}(\vec{k}+\vec{q}, \vec{k})$ formfactor, expressing it in the form of the sum including two addenda:

$$
W_{0}(\vec{k}+\vec{q}, \vec{k})=v_{q}+f(\vec{k}, \vec{q})
$$

In accordance with the condition of electric neutrality of the system:

$$
W_{0}(\vec{k}, \vec{k})=\lim _{q \rightarrow 0}\left(v_{q}+z V_{q}\right)+f(\vec{k}, 0)
$$

The calculation of characteristics of the electron-ionic model with a local interaction is not very difficult [7-9]. As it has been shown in [10], within the perturbation theory with respect to the operator $H_{d}$ the characteristics in this case are presented in the form of the expansions with respect to $n$-particle static correlation function of the many-particle correlation. However, the local model is a rough approach, due to nonlocality of the formfactors $W_{0}(\vec{k}+\vec{q}, \vec{k})$, their dependence on vector $\vec{k}$ is essential and belongs to the principal peculiarities of the metal model $[4,5]$. Nonlocality makes the calculation of the model characteristics (1-4) essentialby more difficult. Inspite the interst to this problem, a largenumber of publications, and different approximate methods ofnonlocality (see [11-13]) taking into account the problem still remains actual.In this paper by the way of generalizing of the methods developed inthe work [10] in the framework of the basic approach, the strictpresentation of the thermodynamical potential for the nonlocalelectron-ionic model in terms of $\vec{k}$-component of $n$ particlecorrelation function of the basic system - electronic liquid, hasbeen obtained. We use the adiabatic approach for ions, although one can work beyond this restriction using the methods presented in [10].

\section{Partition function of the model in the basic approach}

The purpose of this work is the determination of the partition function of model (1) with respect to the electron variables in the grand canonical ensemble:

$$
Z(\mu)=S p\{\exp [-\beta(\hat{H}-\mu \hat{N})]\}=\exp \left\{-\beta \hat{H}_{i}(R)-\beta \Omega(\mu)\right\},
$$


where $\beta=\left(k_{B} T\right)^{-1}$ is the inverse temperature; $\mu$ is the chemical potential; $\hat{N}$ is the operator of the electron number. The model of electron liquid, for which the thermodynamical potential and the correlation function are known [14], has to be used as a basic system. For this let us use the interaction representation [15] for statistical operator

$$
\begin{aligned}
& \exp [-\beta(\hat{H}-\mu \hat{N})]=\exp \left[-\beta \hat{H}_{i}(\vec{R})\right] \exp \left[-\beta\left(\hat{H}_{0}-\mu \hat{N}\right)\right] T\left\{\hat{S}^{e} \hat{S}\right\}, \\
& \hat{S}^{e}=\exp \left\{-\int_{0}^{\beta} \hat{V}_{e e}\left(\beta^{\prime}\right) d \beta^{\prime}\right\}, \quad \hat{S}=\exp \left\{-\int_{0}^{\beta} \hat{H}_{e i}\left(\beta^{\prime}\right) d \beta^{\prime}\right\},
\end{aligned}
$$

where $T$ is the symbol of the usual chronological ordering. The partition function will be presented as

$$
Z(\mu)=\exp \left\{-\beta \hat{H}_{i}(R)\right\} Z_{e}(\mu)<\hat{S}>_{e}=\exp \left\{-\beta \hat{H}_{i}(R)\right\} Z_{0}(\mu)<T\left\{\hat{S}^{e} \hat{S}\right\}>_{0},
$$

where $Z_{0}(\mu)$ denotes the partition function of the ideal electron system, and $Z_{e}(\mu)$ is the partition function for the basic system. Respectively the symbol $\langle\ldots\rangle_{0}$ denotes the statistical average over the ideal system states of electron liquid model and $\langle\ldots\rangle_{e}$ - by the states of the basic one. For more convenient calculation of diagrams of the perturbation theory let us go to the frequency presentation [16], according to which

$$
\begin{gathered}
<T\left\{\hat{S}^{e} \hat{S}\right\}>_{0}=<\tilde{T}\left\{\hat{S}_{\nu}^{e} \hat{S}_{\nu}\right\}>_{0}, \\
\hat{S}_{\nu}^{e}=\exp \left\{-(2 \beta V)^{-1} \sum_{\vec{q} \neq 0} \sum_{\nu} V_{q} \hat{\rho}_{\vec{q}, \nu} \hat{\rho}_{-\vec{q},-\nu}\right\}, \\
\hat{S}_{\nu}=\exp \left\{-N_{i} V^{-1} \sum_{\vec{q}} S_{-\vec{q}} \hat{R}_{\vec{q}}\right\}, \\
\hat{\rho}_{x} \equiv \hat{\rho}_{\vec{q}, \nu}=\sum_{\vec{k}, s} \sum_{\nu^{*}} C_{\vec{k}+\vec{q}, s}^{+}\left(\nu^{*}+\nu\right) C_{\vec{k}, s}\left(\nu^{*}\right) \\
\hat{R}_{\vec{q}}=\sum_{\vec{k}, s} \sum_{\nu^{*}} W_{0}(\vec{k}+\vec{q}, \vec{k}) C_{\vec{k}+\vec{q}, s}^{+}\left(\nu^{*}\right) C_{\vec{k}, s}\left(\nu^{*}\right),
\end{gathered}
$$

where $\nu^{*}=(2 n+1) \pi \beta^{-1}$ are the Fermi-Matsubara frequencies, and $\nu=$ $2 n \pi \beta^{-1}$ are the Bose-Matsubara frequencies [15]; $C_{\vec{k}, s}\left(\nu^{*}\right)$ is the linear conbination of operators $C_{\vec{k}, s}$ in the interaction representation, namely:

$$
C_{\vec{k}, s}\left(\nu^{*}\right)=\beta^{-1 / 2} \int_{0}^{\beta} C_{\vec{k}, s}\left(\beta^{\prime}\right) \exp \left(i \nu^{*} \beta^{\prime}\right) d \beta^{\prime}
$$

Besides it was used the symbol of generalized chronolgical ordering $\tilde{T}$, which possesses the properties of the $T$ symbol and leads the product of $a_{k, s}\left(\beta^{\prime}\right)$ operators to normal form for coinciding arguments [16]. The operators 
$C_{\vec{k}, s}\left(\nu^{*}\right)$ do not possess an independent sense and they would be considered only as a designation. However, they would obtain such a sense while determining the average from the product of the two operators using the experssion:

$$
-\left\langle\tilde{T}\left\{C_{\vec{k}, s_{1}}\left(\nu_{1}^{*}\right) C_{\vec{k}, s_{2}}^{+}\left(\nu_{2}^{*}\right)\right\}\right\rangle_{0}=\delta_{\vec{k}_{1}, \vec{k}_{2}} \delta_{s_{1}, s_{2}} \delta_{\nu_{1}^{*}, \nu_{2}^{*}} G_{\vec{k}_{1}, s_{1}}^{0}\left(\nu_{1}^{*}\right)
$$

where $G_{\vec{k}, s}^{0}\left(\nu^{*}\right)=\left\{i \nu^{*}-\varepsilon_{k}+\mu\right\}^{-1}$ is the spectral representation of the oneparticle Green function for the ideal system. In expression (11) $\hat{\rho}_{x}$ denotes the spectral representation of the electron density operator, and $\hat{R}_{\vec{q}}$ by its structure reminds the statistical limit of such operator, moreover, every $\vec{k}$ - component is included with accounting of their weights $W_{0}(\vec{k}+\vec{q}, \vec{k})$. Expanding $\hat{S}_{\nu}$ operator into the power series, averaging the terms and presenting the result in an exponential form, one can obtain the expression for the thermodynamical potential of the model:

$$
\begin{gathered}
\Omega(\mu)=\Omega_{e}(\mu)+\sum_{n \geqslant 1} \Omega_{n}(\mu) \\
\Omega_{n}(\mu)=(-1)^{n+1} \beta^{-1} N_{i}^{n} V^{-n}(n !)^{-1} \sum_{\vec{q}_{1}, \ldots, \vec{q}_{n}} S_{-\vec{q}_{1}} S_{-\vec{q}_{2}} \ldots S_{-\vec{q}_{n}}\left\langle\hat{R}_{\vec{q}_{1}} \hat{R}_{\vec{q}_{2}} \ldots \hat{R}_{\vec{q}_{n}}\right\rangle_{e}^{c}
\end{gathered}
$$

where $<\ldots>_{e}^{c}$ symbol denotes the bound average of the product of $\hat{R}_{\vec{q}}$ operators and $\Omega_{e}(\mu)$ is the grand thermodynamical potential of the electron liquid. According to (6), (7):

$$
\Omega_{l}(\mu)=N_{i} V^{-1} \sum_{\vec{k}, \vec{s}} n_{\vec{k}, s} W_{0}(\vec{k}, \vec{k}),
$$

where $n_{\vec{k}, s}$ is the electron distribution with respect to the momenta in the basic system. For the calculation of the terms $\Omega_{n}(\mu)$ for $n \geqslant 2$ let us expand the operator $\hat{S}_{\nu}^{e}$ into a power series and average term by term over the states of the ideal system (which is considered as a basic in the model of the electron liquid). In this case there appear the cumulant correlation functions constructed on the operators $\hat{\rho}_{x}[17]$

$$
\tilde{\mu}_{n}^{0}\left(x_{1}, \ldots, x_{n}\right)=\beta^{-1}\left\langle\tilde{T}\left\{\hat{\rho}_{x_{1}} \hat{\rho}_{x_{2}} \ldots \rho_{x_{n}}\right\}\right\rangle_{0}^{c}=\delta_{x_{1}+x_{2}+\ldots+x_{n}, 0} \sum_{\vec{k}} \tilde{\mu}_{n}^{0}\left(x_{1}, \ldots, x_{n} \mid \vec{k}\right) .
$$

Here we introduce $\vec{k}$-component of the n-particle dynamical correlation function of the electron ideal system. By the spherical limits of these $\vec{k}$ components :

$$
\hat{\mu}_{n}^{0}\left(\vec{q}_{1}, \ldots, \vec{q}_{n} \mid \vec{k}\right)=\lim _{\nu_{1}, \ldots, \nu_{n} \rightarrow 0} \tilde{\mu}_{n}^{0}\left(x_{1}, \ldots, x_{n} \mid \vec{k}\right)
$$

are determined the averages, which are built on the operators $\hat{R}_{\vec{q}}$, namely:

$$
\beta^{-1}\left\langle\tilde{T}\left\{\hat{R}_{\vec{q}_{1}} \hat{R}_{\vec{q}_{2}} \cdots \hat{R}_{\vec{q}_{n}}\right\}\right\rangle_{0}^{c}=\delta_{\vec{q}_{1}+\ldots+\vec{q}_{n}, 0} \sum_{\vec{k}} \tilde{\mu}_{n}^{0}\left(\vec{q}_{1}, \ldots, \vec{q}_{n} \mid \vec{k}\right) \times
$$




$$
\times W_{0}\left(\vec{k}+\vec{q}_{1}, \vec{k}\right) W_{0}\left(\vec{k}, \vec{k}-\vec{q}_{2}\right) \cdots W_{0}\left(\vec{k}-\vec{q}_{2}-\cdots-\vec{q}_{n-1}, \vec{k}-\vec{q}_{2}-\cdots-\vec{q}_{n}\right)
$$

as well as the mixed averages like

$$
\begin{aligned}
& \beta^{-1}\left\langle\tilde{T}\left\{\hat{R}_{\vec{q}_{1}} \hat{R}_{\vec{q}_{2}} \cdots \hat{R}_{\vec{q}_{m}} \hat{\rho}_{x_{m=1}} \cdots \hat{\rho}_{x_{n}}\right\}\right\rangle_{0}^{c}=\delta_{\vec{q}_{1}+\ldots+\vec{q}_{n}, 0} \prod_{j=m+1}^{n} \delta_{\nu_{j}, 0} \sum_{\vec{k}} \tilde{\mu}_{n}^{0}\left(\vec{q}_{1}, \ldots \vec{q}_{n} \mid \vec{k}\right) \times \\
& \times W_{0}\left(\vec{k}+\vec{q}_{1}, \vec{k}\right) W_{0}\left(\vec{k}, \vec{k}-\vec{q}_{2}\right) \cdots W_{0}\left(\vec{k}-\vec{q}_{2}-\cdots-q_{m-1}, \vec{k}-\vec{q}_{2}-\cdots-\vec{q}_{m}\right) . \quad(19)
\end{aligned}
$$

Using the methods of the work [10] let us summarize the sets of diagrams of a geometrical progression type, built on the functions (16)-(19) relative to the electron-electron interaction. This allows us to represent the component of $\Omega_{n}(\mu)$ over the $\vec{k}$-components of the $n$-particle static polarization operators of the basic system - the homogenous electron liquid.

$$
M_{n}\left(\vec{q}_{1}, \ldots \vec{q}_{n}\right)=\sum_{\vec{k}} M_{n}\left(\vec{q}_{1}, \ldots, \vec{q}_{n} \mid \vec{k}\right)
$$

The expression for the component of the second order of the perturbation theory may be written as

$$
\begin{gathered}
\Omega_{2}(\mu)=-\frac{N^{2}}{2 ! V^{2}} \sum_{\vec{q}} S_{\vec{q}} S_{-\vec{q}}\left\{F_{22}(\vec{q})-\frac{V_{q}}{V \varepsilon(q)} F_{21}(\vec{q}) F_{21}(-\vec{q})\right\} \\
F_{21}(\vec{q})=\sum_{\vec{k}} M_{2}(\vec{q},-\vec{q} \mid \vec{k}) W_{0}(\vec{k}+\vec{q}, \vec{k}), \\
F_{22}(\vec{q})=\sum_{\vec{k}} M_{2}(\vec{q},-\vec{q} \mid \vec{k}) W_{0}(\vec{k}+\vec{q}, \vec{k}) W_{0}(\vec{k}, \vec{k}+\vec{q}),
\end{gathered}
$$

In this case

$$
\varepsilon(q)=1+\frac{V_{q}}{V} M_{2}(\vec{q},-\vec{q})
$$

is the static dielectric function of the basic system. For the third order we will have:

$$
\begin{aligned}
\Omega_{3}(\mu)=\frac{N_{i}^{3}}{3 ! V^{3}} \sum_{\vec{q}_{1}, \vec{q}_{2}, \vec{q}_{3}} \delta_{\vec{q}_{1}+\vec{q}_{2}+\vec{q}_{3}, 0} S_{\vec{q}_{1}} S_{\vec{q}_{2}} S_{\vec{q}_{3}}\left\{F_{33}\left(\vec{q}_{1}, \vec{q}_{2}, \vec{q}_{3}\right)-\right. \\
\quad-3 F_{32}\left(\vec{q}_{1}, \vec{q}_{2}, \vec{q}_{3}\right) \frac{V_{q_{3}}}{V \varepsilon\left(q_{3}\right)} F_{21}\left(\vec{q}_{3}\right)+ \\
+3 F_{31}\left(\vec{q}_{1}, \vec{q}_{2}, \vec{q}_{3}\right) \prod_{i=1}^{2}\left(V \varepsilon\left(q_{i}\right)\right)^{-1} V_{q_{i}} F_{21}\left(\vec{q}_{i}\right)- \\
\left.-M_{3}\left(\vec{q}_{1}, \vec{q}_{2}, \vec{q}_{3}\right) \prod_{i=1}^{3}\left(V \varepsilon\left(q_{i}\right)\right)^{-1} V_{q_{i}} F_{21}\left(\vec{q}_{i}\right)\right\}
\end{aligned}
$$

Here the follouwing notations have been used:

$$
F_{31}\left(\vec{q}_{1}, \vec{q}_{2}, \vec{q}_{3}\right)=\sum_{\vec{k}} M_{3}\left(\vec{q}_{1}, \vec{q}_{2}, \vec{q}_{3} \mid \vec{k}\right) W_{0}\left(\vec{k}+\vec{q}_{1}, \vec{k}\right),
$$




$$
F_{32}\left(\vec{q}_{1}, \vec{q}_{2}, \vec{q}_{3}\right)=\sum_{\vec{k}} M_{3}\left(\vec{q}_{1}, \vec{q}_{2}, \overrightarrow{q_{3}} \mid \vec{k}\right) W_{0}\left(\vec{k}+\vec{q}_{1}, \vec{k}\right) W_{0}\left(\vec{k}, \vec{k}-\vec{q}_{2}\right),
$$

$F_{33}\left(\vec{q}_{1}, \vec{q}_{2}, \vec{q}_{3}\right)=\sum_{\vec{k}} M_{3}\left(\vec{q}_{1}, \vec{q}_{2}, \overrightarrow{q_{3}} \mid \vec{k}\right) W_{0}\left(\vec{k}+\vec{q}_{1}, \vec{k}\right) W_{0}\left(\vec{k}, \vec{k}-\vec{q}_{2}\right) W_{0}\left(\vec{k}-\overrightarrow{q_{2}}, \vec{k}-\vec{q}_{2}-\vec{q}_{3}\right), \ldots$

The expressions for the components corresponding to $n \geqslant 4$ posses an analogous structure, although we are not going to present them here due to their growing awkward. $\Omega_{n}(\mu)$ at $n \geqslant 2$ may be presented in more compact form. Using the division of $W_{0}(\vec{k}+\vec{q}, \vec{k})$ onto two components (7) and introducing the function:

$$
g(\vec{q})=V_{q}(V \varepsilon(q))^{-1} \sum_{\vec{k}} M_{2}(\vec{q},-\vec{q} \mid \vec{k}) f(\vec{k}, \vec{q}),
$$

one can transform $\Omega_{n}(\mu)$ into the eqvivalent form:

$$
\begin{gathered}
\Omega_{n}(\mu)=(-1)^{n+1} \frac{N_{i}^{n}}{n ! V^{n}} \sum_{\vec{k}} \omega_{n}(\mu \mid \vec{k}) \\
\omega_{2}(\mu \mid \vec{k})=\sum_{\vec{q}} S_{\vec{q}} S_{-\vec{q}} M_{2}(\vec{q},-\vec{q} \mid \vec{k}) W(\vec{k}+\vec{q}, \vec{k}) W(\vec{k}, \vec{k}+\vec{q}) \\
\omega_{n}(\mu \mid \vec{k})=\sum_{\vec{q}_{1}, \ldots \vec{q}_{n}} S_{\vec{q}_{1}} S_{\vec{q}_{2}} \ldots S_{\vec{q}_{n}} \delta_{\vec{q}_{1}+\ldots+\vec{q}_{n}, 0} M_{n}\left(\vec{q}_{1}, \ldots \overrightarrow{q_{n}} \mid \vec{k}\right) \times \\
\quad \times W\left(\vec{k}+\overrightarrow{q_{1}}, \vec{k}\right) W\left(\vec{k}+\overrightarrow{q_{2}}, \vec{k}\right) \ldots W\left(\vec{k}+\vec{q}_{n}, \vec{k}\right)
\end{gathered}
$$

In this case

$$
W(\vec{k}+\vec{q}, \vec{k})=\nu_{q} \varepsilon^{-1}(q)+f(\vec{k}, \vec{q})-g(\vec{q})
$$

is the statically screened nonlocal formfactor. As it follows from (25)-(27), the nonlocal part of the formfactor is also simplified, since $f(\vec{k}, \vec{q})-g(\vec{q})$ as a function of $\vec{q}$-vector possesses the properties of the screened potential:

$$
\begin{gathered}
f(\vec{k}, \vec{q})-g(\vec{q})=\varepsilon^{-1}(q)\langle f(\vec{k}, \vec{q})\rangle_{k}+\left\{f(\vec{k}, \vec{q})-\langle f(\vec{k}, \vec{q})\rangle_{k}\right\} \\
\langle f(\vec{k}, \vec{q})\rangle_{k} \equiv M_{2}^{-1}(q,-q) \sum_{\vec{k}} M_{2}(\vec{q},-\vec{q} \mid \vec{k}) f(\vec{k}, \vec{q})
\end{gathered}
$$

The component in the figured brackets is less when the nonlocality is less, i.e the smoother is the dependence on $\vec{k}$-vector. Therefore, the thermodynamical potential of the electron model with nonlocal interaction generalizes the corresponding representation $\Omega(\mu)$ for the local interaction and is expressed in the form of a single sum over $\vec{k}$-vector.

Now, going to the variables of the canonical ensemble $\beta, V, N$ let us indroduce the free energy of the model:

$$
F=\Omega(\mu *)+N \mu^{*},
$$

where $\mu$ is the chemical potential determined by equation:

$$
N=-\frac{d}{d \mu^{*}} \Omega\left(\mu^{*}\right)
$$


We consider the conditions, under which the deviation $\mu^{*}$ from the chemical potential of the electron liquid model $\mu_{e}$ is as follow:

$$
\mu^{*}=\mu_{e}+\delta \mu,
$$

where $|\delta \mu|<<\left|\mu_{e}\right|$. Subsituting (31) into (30) one can expand $\Omega\left(\mu^{*}\right)$ into the Taylor series in the vicinity of $\mu_{e}$ point. Taking into account that $N=-\frac{d}{d \mu_{e}} \Omega_{e}\left(\mu_{e}\right)$, in the linear approach we will obtain:

$$
\delta \mu=-\left[\frac{d^{2}}{d \mu_{e}^{2}} \Omega_{e}\left(\mu_{e}\right)\right]^{-1} \sum_{n \geqslant 1} \frac{d}{d \mu_{e}} \Omega_{n}\left(\mu_{e}\right)
$$

since

$$
\frac{d^{2}}{d \mu_{e}^{2}} \Omega_{e}\left(\mu_{e}\right)=-M_{2}(0,0)
$$

where $\mathrm{M}_{2}(0,0)$ is the long-wave limit of a pair static polarization operator of the basic system, then in the adopted approach the free energy will be written as:

$$
F=F_{e}+\sum_{n \geqslant 1} \Omega_{n}\left(\mu_{e}\right)+\frac{1}{2 !} M_{2}(0,0)[\delta \mu]^{2}+\ldots
$$

Here $F_{e}=\Omega_{e}\left(\mu_{e}\right)+N \mu_{e}$ is the free energy of the basic system, and the component $\Omega_{n}\left(\mu_{e}\right)$ are determined by the true correlation functionsof the basic system in the variables $\beta, V, N$.

In the approach of a local field the $\vec{k}$-components of the polarization operators of the basic system, involved in $(25)-(26)$, have the same form:

$$
\begin{gathered}
M_{2}(\vec{q},-\vec{q} \mid \vec{k})=\tilde{\mu}_{2}^{0}(\vec{q},-\vec{q} \mid \vec{k})\left\{1-\frac{V_{q}}{V} \tilde{\mu}_{2}^{0}(\vec{q},-\vec{q}) G(q)\right\}^{-1}, \\
M_{n}\left(\vec{q}_{1}, \ldots \vec{q}_{n} \mid \vec{k}=\tilde{\mu}_{n}^{0}\left(\vec{q}, \ldots, \vec{q}_{n} \mid \vec{k}\right) \prod_{i=1}^{n}\left\{1-\frac{V_{q_{i}}}{V} \tilde{\mu}_{2}^{0}\left(\vec{q}_{i},-\vec{q}_{i}\right) G\left(q_{i}\right)\right\}^{-1},\right.
\end{gathered}
$$

where $G\left(q_{i}\right)$ is the correction due to local field in the static limit (see, for example [14]). According to (13)-(16):

$$
\tilde{\mu}_{n}^{0}\left(\vec{q}_{1}, \ldots, \vec{q}_{n} \mid \vec{k}\right)=(-1)^{n+1}(\beta n)^{-1} \delta_{\vec{q}_{1}+\ldots+\vec{q}_{n}, 0} \sum_{i_{1} \neq i_{2} \neq \ldots \neq i_{n}=1}^{n} \sum_{s} \sum_{\nu^{*}} \prod_{j=1}^{n} G_{\vec{k}-\vec{\chi}_{j}, s}^{0}\left(\nu^{*}\right),
$$

where $\vec{\chi}_{j}=\sum_{l=1}^{j} \vec{q}_{j_{l}}$. Summarizing over the $\nu^{*}$ frequency, we will obtain the representation of $\tilde{\mu}_{n}^{0}\left(\vec{q}_{1}, \ldots, \vec{q}_{n} \mid \vec{k}\right)$ in the form of linear combination of the elements of the following type:

$$
\gamma_{n}\left(\vec{q}_{1}, \ldots, \vec{q}_{n-1} \mid \vec{k}\right)=-2 \sum_{s} n_{\vec{k}, s}^{0} \prod_{r=1}^{n-1}\left\{\varepsilon_{\vec{k}}-\varepsilon_{\vec{k}+\vec{q}_{r}}\right\}^{-1} .
$$

In the cases of degeneration (when together with the condition $\vec{q}_{1}+\vec{q}_{2}+$ $\ldots+\vec{q}_{n}=0$ the condition $\vec{q}_{1}+\vec{q}_{2}+\ldots+\vec{q}_{j}=0$ also is fulfilled at $2 \leqslant \mathrm{~J} \leqslant n-2$ ) 
beside the functions $\gamma_{n}\left(\vec{q}_{1}, \ldots, \vec{q}_{n-1}\right)$ there are appear also the lower order derivatives of the functions (37) with respect to the chemical potential, that is:

$$
\frac{d^{l}}{d \mu^{l}} \gamma_{m}\left(\vec{q}_{1}, \ldots, \vec{q}_{m-1} \mid \vec{k}\right)=-2 \sum_{s} \frac{d^{l} n_{\vec{k}, s}^{0}}{d \mu^{l}} \prod_{r=1}^{m-1}\left\{\varepsilon_{\vec{k}}-\varepsilon_{\vec{k}+\vec{q}_{r}}\right\}^{-1}
$$

where $l+m=n, \quad 1 \leqslant l \leqslant \frac{n}{2}-1$. In the expressions (37)-(38) $n_{\vec{k}, s}^{0}$ denotes the distribution of electrons by momenta in an ideal system. In terms of the work $[16], \gamma_{n}(\ldots)$ are the "normal", and $\frac{d^{l}}{d \mu^{l}} \gamma_{m}(\ldots)$ "anomalous" components of $\vec{k}$-component correlation function of the ideal electron gas. In accordance with expressions (36)-(38) :

$$
\begin{gathered}
\tilde{\mu}_{2}^{0}(\vec{q},-\vec{q} \mid \vec{k})=-2 \sum_{s} n_{\vec{k}, s}^{0}\left(\varepsilon_{\vec{k}}-\varepsilon_{\vec{k}+\vec{q}}\right)^{-1} ; \\
\tilde{\mu}_{3}^{0}\left(\vec{q}_{1}, \vec{q}_{2}, \overrightarrow{q_{3}} \mid \vec{k}\right)=\delta_{\vec{q}_{1}+\vec{q}_{2}+\vec{q}_{3}, 0}\left\{\gamma_{3}\left(\vec{q}_{1},-\vec{q}_{2} \mid \vec{k}\right)+\gamma_{3}\left(\vec{q}_{2},-\vec{q}_{3} \mid \vec{k}\right)+\gamma_{3}\left(\vec{q}_{3},-\vec{q}_{1} \mid \vec{k}\right)\right\}, \\
\gamma_{3}\left(\vec{q}_{1}, \vec{q}_{2} \mid \vec{k}\right)=2 \sum_{s} n_{\vec{k}, s}^{0}\left(\varepsilon_{\vec{k}}-\varepsilon_{\vec{k}+\vec{q}_{1}}\right)^{-1}\left(\varepsilon_{\vec{k}}-\varepsilon_{\vec{k}+\vec{q}_{2}}\right)^{-1}, \\
\tilde{\mu}_{4}^{0}\left(\vec{q}_{1},-\vec{q}_{1}, \vec{q}_{2},-\vec{q}_{2} \mid \vec{k}\right)=\sum_{\sigma= \pm 1}\left\{\gamma_{4}\left(\vec{q}_{1}, \vec{q}_{1}, \vec{q}_{1}+\sigma \vec{q}_{2} \mid \vec{k}\right)-\gamma_{4}\left(\vec{q}_{1}, \vec{q}_{1}, \sigma \vec{q}_{2} \mid \vec{k}\right)\right. \\
+\gamma_{4}\left(\vec{q}_{2}, \vec{q}_{2}, \vec{q}_{2}+\sigma \vec{q}_{1} \mid \vec{k}\right)-\gamma_{4}\left(\vec{q}_{2}, \vec{q}_{2}, \sigma \vec{q}_{1} \mid \vec{k}\right)+2 m \hbar^{-2} \sigma\left(\vec{q}_{1} \vec{q}_{2}\right)^{-1}\left[\gamma_{3}\left(\vec{q}_{1}, \vec{q}_{1}+\sigma \vec{q}_{2} \mid k\right)\right. \\
\left.\left.+\gamma_{3}\left(\vec{q}_{2}, \vec{q}_{2}+\sigma \vec{q}_{2} \mid \vec{k}\right)-\gamma_{3}\left(\vec{q}_{1}, \sigma \vec{q}_{2} \mid \vec{k}\right)\right]\right\}-\sum_{\sigma= \pm 1} \frac{d}{d \mu} \gamma_{3}\left(\vec{q}_{1}, \sigma \vec{q}_{2} \mid \vec{k}\right), \\
\gamma_{4}\left(\vec{q}_{1}, \vec{q}_{1}, \vec{q}_{2} \mid \vec{k}\right)=-2 \sum_{s} n_{\vec{k}, s}^{0}\left(\varepsilon_{\vec{k}}-\varepsilon_{\vec{k}+\vec{q}_{1}}\right)^{-2}\left(\varepsilon_{\vec{k}}-\varepsilon_{\vec{k}+\vec{q}_{2}}\right)^{-1} .
\end{gathered}
$$

Therefore, the functions $\tilde{\mu}_{n}^{0}\left(\vec{q}_{1}, \ldots, \vec{q}_{n} \mid \vec{k}\right)$ of any order are presented in an analytical form due to which the calculation of $\Omega_{n}\left(\mu_{e}\right)$ reduced to the computation of the multiple integrals in a space of the wave vectors.

\section{References}

[1] Heine V., Cohen M., Weaire D. The Pseudopetential Concept. Moscow, Mir, 1973 (in Russian).

[2] Harrison W.A. Pseudopotential in the theory of metals. New York, W.A.Benjamin, 1966.

[3] Vavrukh M., Krokhmalskii T. Many particle nonlocal interaction of electrons with ions in metals. // Phys. of Metals and Metal Sci. (FMMS), 1985, vol. 59, No 5, p. 864-874 (in Russian).

[4] Vavrukh M., Muliava Y. The localizel electron state taking into account in the microscopic theory of metals. // JPS, 1996, vol. 1, No 2, p. 1-10 (in Ukrainian).

[5] Dutchak Y.I., Yakibchuk P.M., Zhovtanetckii M.I. Binding energy and equilibrium atomic radiusses of nontransition metals within the model pseudopotential method. // Ukr. Fiz. Zhurn., 1976, vol. 21, No 1, p. ?-? (in Ukrainian). 
[6] Fourman V.V., Yakibchuk P.M. Pseudopotential within the phase functions metod. Structure of model pseudopotential of transition and rare-earth metals. // JPS, 1996, vol. 1, No 1, p. 134-137 (in Ukrainian).

[7] Browman E.G., Kagan Y.M. Phonons in nontransition metals. // Usp. Fiz. Nauk, 1974, vol. 112, No 3, p. 369-426 (in Russian).

[8] Gurskii Z. A., Krasko G.A. Model pseudopotential and stability of crystal structures of some simple metals. // Solid State Phys., 1969, vol. 11, No 10, p. 3016-3019 (in Russian).

[9] Bykov O.Y., Shvets V.T. Effective pair-wise interionic interection in alkali metals. // Ukr. Fiz. Zhurn., 1991, vol. 36, No 3, p. 469-471 (in Ukrainian).

[10] Vavrukh M., Krokhmalskii T. Effective many particle ion interactions in metals. // Teor. Mat. Fiz., 1982, vol. 51, No 1, p. 130-141 (in Russian).

[11] Varsovskij M.I., Kathnelrou M., Trefilov A.V. Electrons localized and delocalized behavior in metals. // Phys. Metals and Metal Sci. (FMMS), 1993, vol. 76, No 3, p. 3-89 (in Russian).

[12] Dutchak Ya.I., Yakibchuk P.M., Baryljak G.I. To the pseudopotential nonlocality effect on the results of bindiry enegry and equilibrium rating lithium calculation. // Phys. Metals and Metal Sci. (FMMS), 1977, vol. 44, No 4, p. 895-896 (in Russian).

[13] Vavrukh M., Solovyan V.B. Dynamical correction on local field of interalting electron gas. // Ukr. Fiz. Zhurn., 1993, vol. 38, No 6, p. 948-954 (in Ukrainian).

[14] Abrikosov A.A., Gorkov L.P., Dzyaloshinskii J.E. Methods of quantum field theory in statistical physycs. Moscow, Fizmatgiz, 1962 (in Russian).

[15] Vavrukh M., Krokhmalskii T. Reference system approach in the electron liquid theory and general relations. // Phys. Stat. Sol. (b), 1991, vol. 162, No 2, p. $519-532$.

[16] Vavrukh M. n-body correlation function of interalting electron gas. // Teor. Mat. Fiz. 1982, vol. 50, No 3, p. 438-449 (in Russian).

\title{
РОЗРАХУНОК ТЕРМОДІНАМІЧНОГО ПОТЕНЦІАЛУ ЕЛЕКТРОНО-ІОННОЇ МОДЕЛІ МЕТАЛУ ІЗ НЕЛОКАЛЬНИМИ ВЗАЕМОДІЯМИ
}

\author{
П.М.Якібчук
}

Термодинамічний потенціал електрон-іонної моделі неперехідних металів 3 двочастинковою нелокальною взаємодією зображено у вигляді розкладів теорії збурень в термінах $n$-частинкових статичних кореляційних функцій та $n$-частинкових поляризаційних операторів однорідної електронної рідини. 